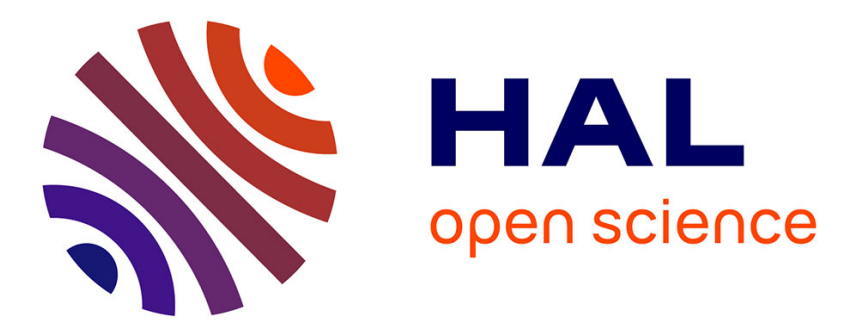

\title{
Modélisation du champ de capteurs de la centrale solaire de vignola
}

\author{
J.H. Balbi, N. Balbi, P. Orenga, G. Simonnot
}

\section{To cite this version:}

J.H. Balbi, N. Balbi, P. Orenga, G. Simonnot. Modélisation du champ de capteurs de la centrale solaire de vignola. Revue de Physique Appliquée, 1986, 21 (2), pp.169-180. 10.1051/rphysap:01986002102016900 . jpa-00245422

\section{HAL Id: jpa-00245422 https://hal.science/jpa-00245422}

Submitted on 1 Jan 1986

HAL is a multi-disciplinary open access archive for the deposit and dissemination of scientific research documents, whether they are published or not. The documents may come from teaching and research institutions in France or abroad, or from public or private research centers.
L'archive ouverte pluridisciplinaire HAL, est destinée au dépôt et à la diffusion de documents scientifiques de niveau recherche, publiés ou non, émanant des établissements d'enseignement et de recherche français ou étrangers, des laboratoires publics ou privés. 


\title{
Modélisation du champ de capteurs de la centrale solaire de vignola
}

\author{
J. H. Balbi, N. Balbi, P. Orenga et G. Simonnot \\ avec la collaboration de G. Girolami, A. Louche, G. Peri et C. Riolacci \\ Université de Corse, Laboratoire d'Hélioénergétique, Route des Sanguinaires, Vignola, 20000 Ajaccio, France
}

(Reçu le 18 mars 1985, révisé le 15 juillet, accepté le 5 novembre 1985)

\begin{abstract}
Résumé. - On construit un modèle de ce système énergétique de taille industrielle. Dans ce but, on soumet le champ à un échelon de flux solaire (défocalisation des chaudières) et l'on dépouille sa réponse en utilisant l'analyse spectrale. Ceci conduit à une simple équation différentielle du second ordre qui représente très correctement la température en sortie de champ. La simplicité de ce modèle est primordiale pour une commande adaptée.
\end{abstract}

\begin{abstract}
We build one model for this large size energetic system. In this aim, we make a solar step (defocalisation of the collectors) and observe the response by use of spectral analysis. It leads to a simple differential equation of second order which gives a very good approximation of output temperature. The simplicity of this model is fundamental for on appropriate command.
\end{abstract}

\section{Introduction.}

Le but à long terme de nos recherches consiste à réaliser le fonctionnement optimal des systèmes énergétiques. La structure et la commande de ces systèmes sont les éléments fondamentaux de ce fonctionnement, mais sur une mauvaise structure il est difficile de réaliser une commande adaptée.

Notre démarche est donc la suivante :

0) On part d'un prototype déjà réalisé.

1) On supprime les commandes, de manière à accéder à l'évolution naturelle, ou libre, du système (système rendu passif). Ainsi les paramètres de la structure, qui en régime de commande sont variables, deviennent constants.

2) On soumet la structure à des excitations types et l'on mesure expérimentalement la réponse (pour un jeu de paramètres constants).

3) Par utilisation de l'Analyse Spectrale on construit à partir de ces réponses un modèle d'état linéaire, caractérisé par une structure mathématique et par les coefficients de celle-ci. Ce modèle n'est valable, pour lors, que pour le jeu de paramètres choisis.

4) On se donne d'autres jeux de paramètres (constants) et l'on refait l'identification du modèle. On arrive avec quelques expériences, et en utilisant les lois physiques qui régissent le système, à déterminer les lois de dépendance des coefficients par rapport aux paramètres. Le modèle est alors complet et permet de prévoir la réponse du système pour des excitations extérieures quelconques et pour des variations arbitraires, mais connues, des paramètres de structure.

5) L'analyse du comportement du système et éventuellement des sous-systèmes permet de faire des modifications de la structure nécessaire à un fonctionnement plus satisfaisant (du système passif).

6) Le système passif étant optimisé et modélisé, on s'intéresse alors à la gestion c'est-à-dire à la commande de certains paramètres de structure. Etant donné que la plupart des systèmes énergétiques comportent des retards nous nous orientons vers une commande prévisionnelle; celle-ci grâce à l'utilisation en temps réel du modèle permet de corriger les variations des sollicitations extérieures par des variations correspondantes des paramètres de structure de telle manière que la réponse du système soit celle désirée.

7) Il est possible que dans la réalité, il faille ensuite superposer une commande par contre-réaction; mais pour l'instant nous ne nous plaçons pas dans cette optique.

Dans le présent travail nous appliquons cette démarche à un système particulier, mais seulement jusqu'à la phase 3. Les phases suivantes feront l'objet d'une publication ultérieure (qui est en voie d'achèvement).

Nous allons donc dans cet article fournir un modèle d'état linéaire pour le Champ des Capteurs de la Centrale Solaire de Vignola, pour un jeu de paramètres constants. Dans la publication suivante nous établirons le modèle complet du champ (paramètres variables); puis nous montrerons que la commande 
actuelle de la Centrale (par feed back) conduit à une mauvaise gestion et nous réaliserons une commande prévisionnelle.

La qualité essentielle demandée au modèle est la simplicité afin de permettre l'insertion de celui-ci (et son utilisation en temps réel) dans un programme de commande. De ce fait nous écartons les approches de type local-numérique (équations locales, intégration numérique) car elles sont toujours très lourdes. Un tel modèle a d'ailleurs été réalisé pour la Centrale de. Vignola (Réf. [1]), néanmoins il ne semble pas donner une bonne représentation du comportement dynamique de l'ensemble du champ (Réf. [2]). Pour notre part nous utilisons la voie qui a été tracée par notre équipe Réf. [3]) et qui a déjà été illustrée sur un système particulier (Réf. [4]). On peut la qualifier de : globale, analytique, dynamico-phénoménologique, en effet :

- elle fournit le comportement global du système,

- elle conduit à des équations différentielles pouvant être résolues analytiquement,

- elle traduit à la fois le comportement dynamique et la physique du système.

Il est à remarquer que les coefficients intervenant dans les équations différentielles sont déterminés expérimentalement lors de l'établissement du système et qu'il n'est donc pas utile de le recaler.

Nous proposons deux modèles représentant la température de sortie du champ de capteurs. Le plus élaboré se résume à une équation différentielle du second ordre.

On peut apprécier dès à présent le degré de simplicité de représentation d'un système aussi complexe; ce résultat est d'autant plus remarquable qu'il est obtenu à partir d'une seule expérience : réponse à un échelon solaire.

\section{Présentation de la centrale et de l'expérimentation.}

L'installation de Vignola est de type thermique à collecteurs distribués. La chaleur produite est utilisée par 2 boucles de conversion (Réf. [5]), pour la production d'électricité directement réinjectée dans le réseau EDF basse tension (puissance unitaire, $50 \mathrm{~kW}$ électriques).

Pour pallier une insuffisance d'ensoleillement ou pour différer la production, une capacité thermique est branchée en parallèle sur l'installation entre le champ de capteurs et les boucles de conversion. Le réservoir qui constitue cette capacité contient 40 tonnes de fluide caloporteur (therphényl-hydrogéné-dénomination commerciale Gilotherm P. W.). Il est de type à chaleur sensible et température stratifiée $\left(180^{\circ} \mathrm{C}\right.$ dans la partie basse, $250^{\circ} \mathrm{C}$ dans la partie haute). A pleine charge, il doit assurer le fonctionnement pendant 2 heures et demie des boucles délivrant une puissance électrique de $79 \mathrm{~kW}$.

Le champ de capteurs C.O.S.S. (Réf. [6]) (capteur optique à secteurs segmentés) est composé de 8 rangées de 48 mètres chacune.
Chaque rangée est constituée de blocs-miroirs et d'une ligne de chaudière (dans laquelle circule le fluide caloporteur). Les rangées couplées en séries forment quatre épingles (voir figure ci-dessous).

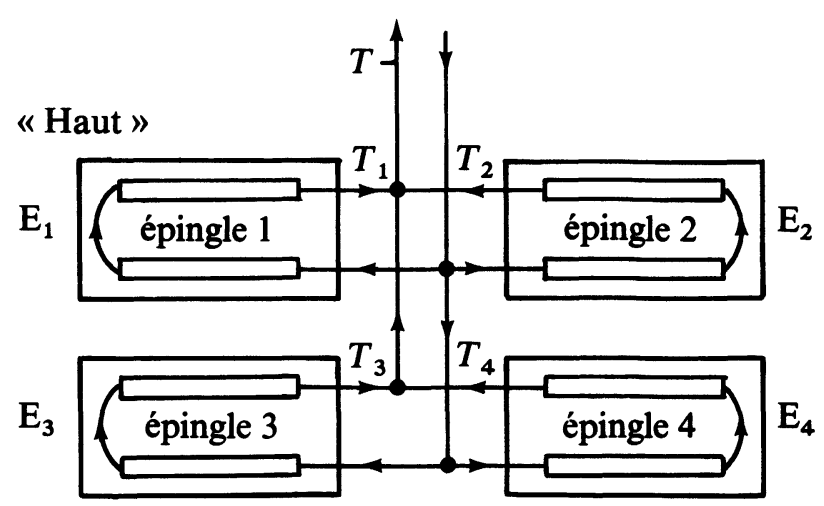

« Bas »

Les blocs miroirs sont fixes. Ils concentrent le rayonnement solaire sur le collecteur (chaudière) où l'énergie solaire est transformée en énergie thermique extraite par le fluide caloporteur.

Les chaudières sont maintenues en position par des bras mobiles. Elles se déplacent sur une surface cylindrique, lieu géométrique de la ligne de focalisation du rayonnement solaire; le maintien en position de focalisation est assuré par un automatisme. La connaissance de l'état de la centrale en fonctionnement et de son environnement a demandé l'installation de capteurs de mesures spécifiques à l'expérimentation (55 au total). Sur le réseau gilotherm, les capteurs de température sont des sondes platine, les débimètres sont à turbine. Nous pouvons connaître les températures aux entrées et aux sorties de chaque rangée, du champ de capteur, du réservoir de stockage et des boucles de conversion ainsi que les débits correspondants. Une installation météorologique fournit les valeurs du rayonnement solaire direct et du rayonnement solaire global horizontal ainsi que la température ambiante.

Tous ces paramètres sont collectés, traités puis stockés sur bande magnétique par une centrale d'acquisition (type 3054 A de Hewlett-Packard). Pour les mesures nécessaires à cet exposé, nous n'avons utilisé que 10 points répartis sur le champ de capteur. Ces 10 points (températures et débits) sont scrutés successivement. Les signaux électriques sont immédiatement traités puis stockés sur bande magnétique. Ces séquences se renouvellent à la cadence de 1 toutes les 4 secondes. La capacité mémoire et la configuration des bandes magnétiques est suffisante pour assurer un fonctionnement sans interruption pendant 1 heure et demie.

\section{Principes de modélisation.}

3. 1 HYPOTHÈSE FONDAMENTALE. - Rappelons l'hypothèse de base qui guide la démarche générale que nous avons proposée (Réf. [3]) : l'E.L.O.D.I.E. (équation linéaire ordinaire différentielle d'état) a été retenue 
comme structure mathématique de base pour sa simplicité et sa généralité.

Le système, rendu passif, est décomposable en soussystèmes dont le modèle est l'E.L.O.D.I.E. Ceux-ci sont reliés entre eux par des relations fonctionnelles à déterminer.

On rend le système passif en supprimant tout ou partie de la commande afin que les paramètres de structure soient constants.

Dans notre cas, où en fonctionnement normal le débit est régulé pour obtenir une température de sortie constante, on supprime cette commande de telle sorte que le débit soit fixe. La température de sortie est alors variable.

\subsection{OBTENTION DU MODÈLE PAR RÉPONSE À UNE ENTRÉE} TYPIQUE. - Pour de tels systèmes énergétiques les grandeurs caractéristiques sont les temps de réponses; pour y accéder un moyen simple est de réaliser un échelon du flux énergétique solaire (il suffit pour celà de procéder à une défocalisation totale ou partielle des chaudières). Par contre, on imagine mal la réalisation d'autres excitations (sinusoïdales par exemple) d'autant plus qu'elles ne sont pas particulièrement bien adaptées pour accéder à des temps de réponse. Un autre avantage de l'échelon de flux comme entrée typique est qu'il correspond assez bien à la réalité de l'évolution du flux lors de passages nuageux.

Nous soumettons donc le champ de capteurs à une défocalisation simultanée et rapide des huit chaudières.

Cette unique et simple expérience de défocalisation est suffisante pour modéliser le système. La figure 1 montre la réponse de la température $T$ de sortie du champ en fonction du temps. Elle évolue de la température d'équilibre initial $T_{\mathrm{f}}$ quand le champ était

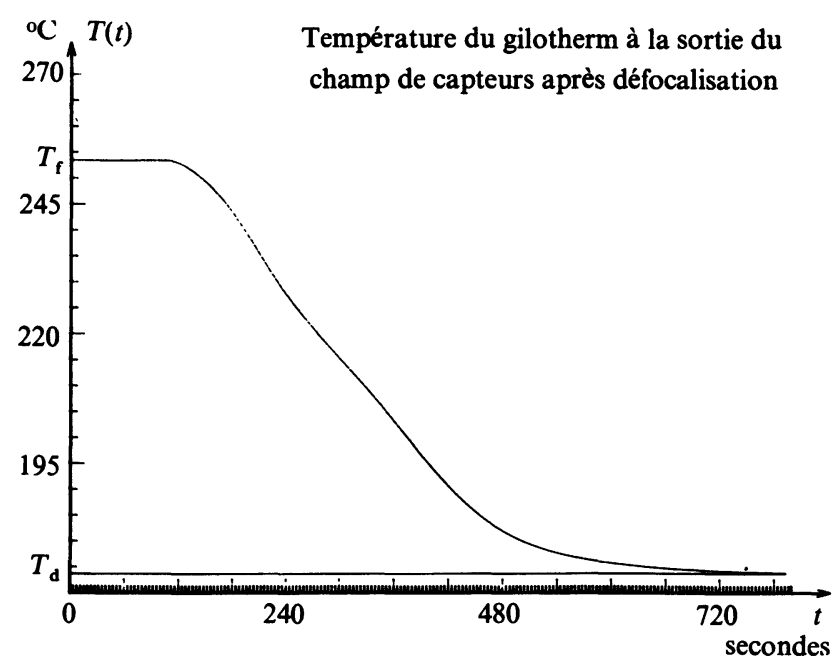

Fig. 1. - Expérience fondamentale : réponse du champ de capteurs à créneau de flux solaire (défocalisation totale).

[Fundamental experimentation : response of the collectors field to a solar flux step (total defocalization).] focalisé, à la température d'équilibre final $T_{\mathrm{d}}$ quand le champ est défocalisé. L'origine des temps correspond à l'instant de défocalisation. C'est une utilisation adéquate de cette courbe fondamentale qui fournit le modèle.

3.3 Premiers essais de modélisation. - Dans un but pédagogique, nous mentionnons ici différents essais de modélisation (bien que ceux-ci n'aient pas été retenus), qui s'incluent dans une démarche logique et systématique de recherche des modèles simples.

En particulier il est naturel de commencer par considérer le système comme une boîte noire, c'està-dire sans le décomposer en sous-systèmes. On élimine ainsi la recherche de relations fonctionnelles souvent difficiles.

3.3. 1 Représentation par une seule E.L.O.D.I.E. - Si le système peut être représenté par une seule E.L.O.D.I.E., la réponse à l'échelon c'est-à-dire le régime libre, doit se traduire par une somme d'exponentielles à coefficients réels négatifs.

$$
\begin{array}{r}
T(t)=A_{0}+A_{1} \exp \left(-\alpha_{1} t\right)+A_{2} \exp \left(-\alpha_{2} t\right)+\cdots \\
\text { avec } \alpha_{1}<\alpha_{2}<\alpha_{3} \cdots .
\end{array}
$$

La valeur constante est immédiatement obtenue, c'est la valeur d'équilibre $T_{\mathrm{d}}$. Pour accéder à la première exponentielle, qui correspond au plus grand temps de réponse, il suffit de tracer $\log \left(T-T_{d}\right)$ et de se placer aux grandes valeurs de $t$ :

$$
\log \left(T-T_{\mathrm{d}}\right) \simeq-\alpha_{1} t+\log A_{1} .
$$

On constate sur la figure 2, qu'il en est bien ainsi ; la fin de la courbe est parfaitement rectiligne; on accède au coefficient de l'exponentielle

$$
\alpha_{1}=0,048(4 \mathrm{~s})^{-1} \text {. }
$$

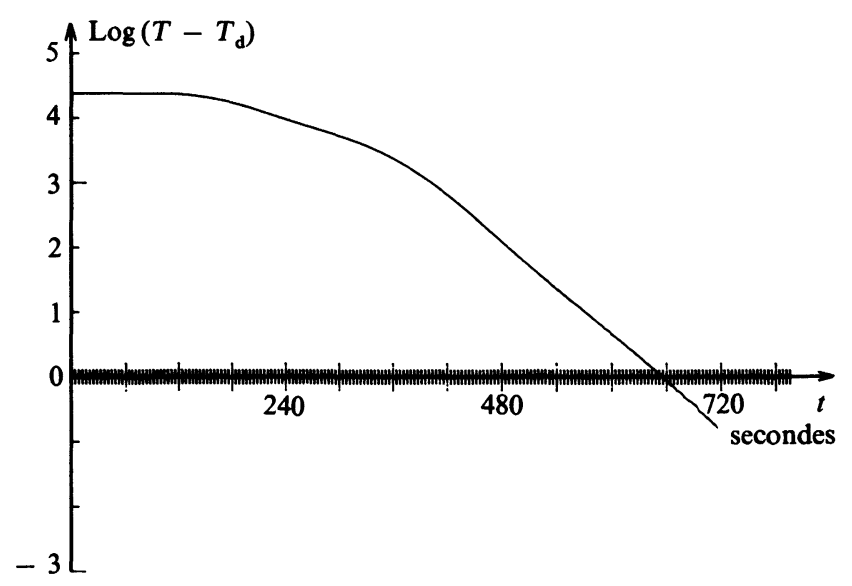

Fig. 2. - Recherche de la première valeur propre par linéarisation logarithmique.

[Research of the first eigenvalue by logarithmic linearization.] 
On continue ce processus de recherches en considérant maintenant

$$
\begin{aligned}
T-\left(T_{\mathrm{d}}\right. & \left.+A_{1} \exp \left(-\alpha_{1} t\right)\right)= \\
& =A_{2} \exp \left(-\alpha_{2} t\right)+A_{3} \exp \left(-\alpha_{3} t\right)+\cdots .
\end{aligned}
$$

Si l'on prend de nouveau le logarithme de cette expression et que l'on regarde la partie extrême droite de la courbe (grandes valeurs de $t$ ) l'on devrait avoir :

$$
\begin{aligned}
\log \left[T-\left(T_{\mathrm{d}}+A_{1} \exp \left(-\alpha_{1} t\right)\right)\right] & \simeq \\
& \simeq-\alpha_{2} t+\log A_{2} .
\end{aligned}
$$

Or, figure 3, il n'apparaît cette fois aucun segment de droite. Il faut donc renoncer à cet essai.

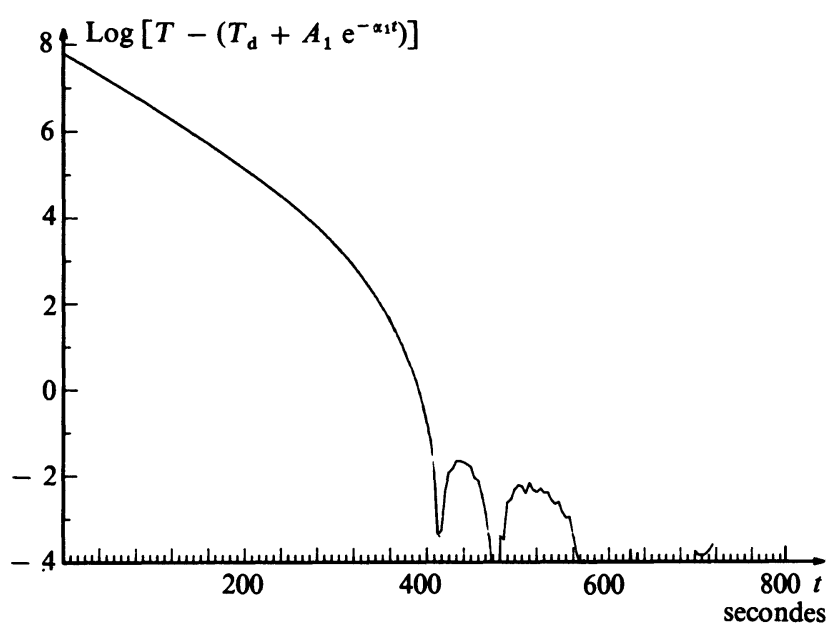

Fig. 3. - Recherche de la deuxième valeur propre par linéarisation logarithmique.

[Research of the second eigenvalue by logarithmic linearization.]

3.3.2 Pseudo-modélisation. - Si l'on revient à la figure 2 , outre la partie linéaire à l'extrême droite déjà signalée, on constate l'existence d'une deuxième partie linéaire. On peut donc représenter $T(t)$ par une exponentielle, mais sur un intervalle limité de temps. Plus précisément :

$$
\begin{aligned}
& T(t)=T_{\mathrm{d}}+A_{1} \exp \left(-\alpha_{1} t\right) \text { pour } t>t_{1} \\
& T(t)=T_{\mathrm{d}}+B \exp (-\beta t) \text { pour } t_{0}<t_{1} .
\end{aligned}
$$

Cette manière de procéder donne très simplement une bonne approximation de la réponse expérimentale (Fig. 4), mais c'est là un bon exemple des pièges de la modélisation : cette approche, malgré son attrait apparent, est inopérante; elle ne conduit pas, en particulier, à une E.L.O.D.I.E.

3.3.3 Modélisation par deux exponentielles décalées. - Le premier essai a fait apparaître que l'on ne pouvait pas, valablement, prolonger la première expo-

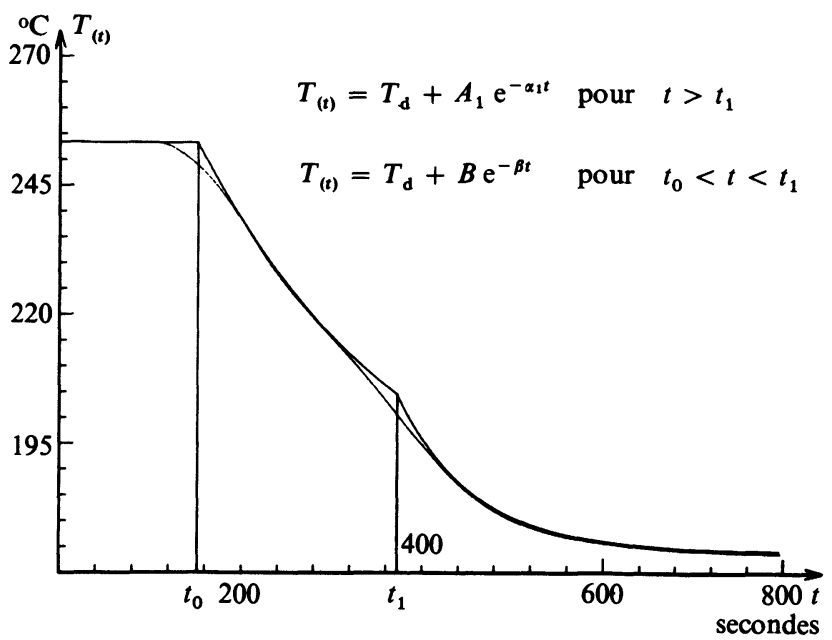

Fig. 4. - Pseudomodélisation par deux exponentielles.

[Pseudo-modelization by use of two exponentials.]

nentielle jusqu'au début de la courbe, car on était alors amené à des valeurs numériques excessives. La physique du problème nous suggère une modélisation du type deux exponentielles à coefficients réels négatifs, décalées dans le temps. On cherche donc $T$ sous la forme :

$$
\begin{gathered}
T(t)=A_{0}+\Gamma\left(t-\tau_{1}\right) A_{1} \exp \left(-\alpha_{1}\left(t-\tau_{1}\right)\right)+ \\
+\Gamma\left(t-\tau_{2}\right) A_{2} \exp \left(-\alpha_{2}\left(t-\tau_{2}\right)\right) \\
\alpha_{1}>0 \text { et } \alpha_{2}>0
\end{gathered}
$$

ou $\Gamma(x)$ est la fonction échelon (nulle pour $x<0$, égale à un pour $x>0$ ).

La procédure de détermination des coefficients devient plus complexe : on introduit de plus les temps de retard. Aussi la solution ne sera pas unique et la méthode pas systématique. On voit ainsi, figure 5, une

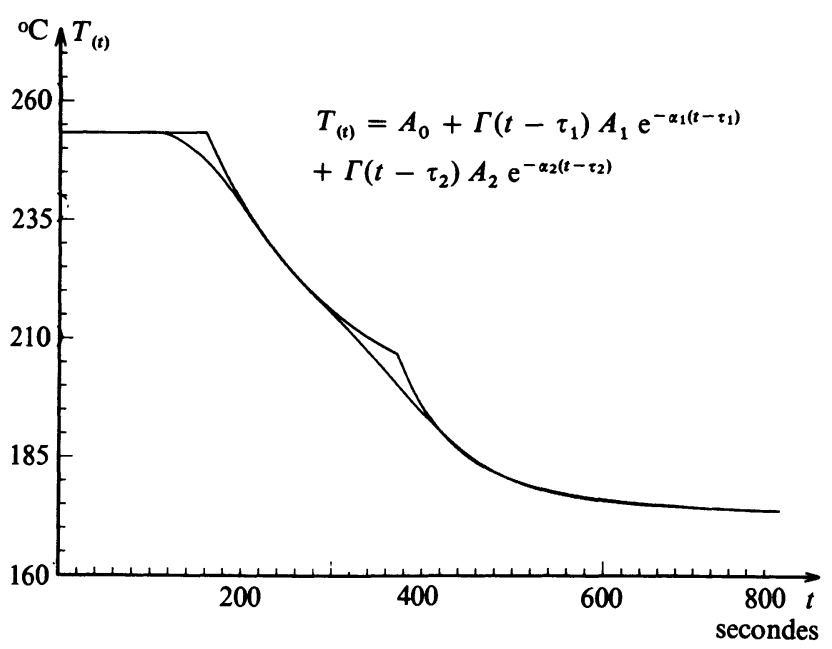

Fig. 5. - Modélisation par deux exponentielles décalées. [Modelization using two delayed exponentials.] 
bonne approximation de $T(t)$ pour un développement à deux exponentielles retardées.

Par ailleurs, cette procédure comporte un autre désavantage : on ne peut pas donner une interprétation physique aux coefficients obtenus, on obtient seulement l'aspect dynamique, ce qui peut conduire à des impasses dans l'utilisation ultérieure du modèle.

3.4 PARTITION EN SOUS-SYSTÈMES. - Pour se rattacher à l'aspect phénoménologique et à la logique de notre démarche, on définit des sous-systèmes modélisables par des E.L.O.D.I.E. Le champ est structuré en quatre épingles : deux «hautes » $E_{1}, E_{2}$ et deux «basses" $E_{3}, E_{4}$ (les dénominations hautes et basses provenant de la situation géographique de la centrale qui est en espaliers). Elles sont pratiquement identiques : si l'ensoleillement est le même sur tout le champ, les températures en sortie d'épingle sont égales.

Compte tenu de la géométrie du système et si l'on suppose négligeables les pertes dans les canalisations entre les points de mesure de température de sortie d'épingle et le point de mesure de $T$, il vient :

$$
\begin{aligned}
T(t)=\frac{1}{4}\left[T_{1}\left(t-\tau_{\mathrm{H}}\right)+\right. & T_{2}\left(t-\tau_{\mathrm{H}}\right)+ \\
& \left.+T_{3}\left(t-\tau_{\mathrm{B}}\right)+T_{4}\left(t-\tau_{\mathrm{B}}\right)\right]
\end{aligned}
$$

en appelant $T_{i}$ les températures en sortie de l'épingle $\mathrm{E}_{i}$ et respectivement $\tau_{\mathbf{H}}$ et $\tau_{\mathbf{B}}$ les temps de parcours nécessaires pour aller de la sortie des épingles respectivement hautes et basses au point de mesure de $T(t)$.

On introduit les températures "d'épingles hautes et basses " :

$$
\begin{aligned}
& T_{\mathrm{H}}(t)=\frac{1}{2}\left[T_{1}\left(t-\tau_{\mathrm{H}}\right)+T_{2}\left(t-\tau_{\mathrm{H}}\right)\right] \\
& T_{\mathbf{B}}(t)=\frac{1}{2}\left[T_{3}\left(t-\tau_{\mathrm{B}}\right)+T_{4}\left(t-\tau_{\mathrm{B}}\right)\right] .
\end{aligned}
$$

Si l'ensoleillement est partout le même

$$
T_{\mathrm{H}}(t)=T_{1}\left(t-\tau_{\mathrm{H}}\right) \quad \text { et } \quad T_{\mathrm{B}}(t)=T_{3}\left(t-\tau_{\mathrm{B}}\right)
$$

alors

$$
T(t)=\frac{T_{\mathrm{H}}(t)+T_{\mathrm{B}}(t)}{2} .
$$

Cette modélisation est très proche de la réalité, en effet si l'on prend l'exemple de la défocalisation, on constate sur les figures 6 et 6 bis que la demi-somme des températures d'épingles convenablement décalées est voisine de la réponse $T(t)$ du champ.

Ce type de relation fonctionnelle régularise les courbes : $T_{1}(t)$ et $T_{3}(t)$ sont très « heurtées " (on distingue très bien le passage dans la première puis seconde chaudière) alors que leur demi-somme l'est beaucoup moins.

Nous retiendrons donc cette formule (1) comme relation fonctionnelle entre le système total et les sous-

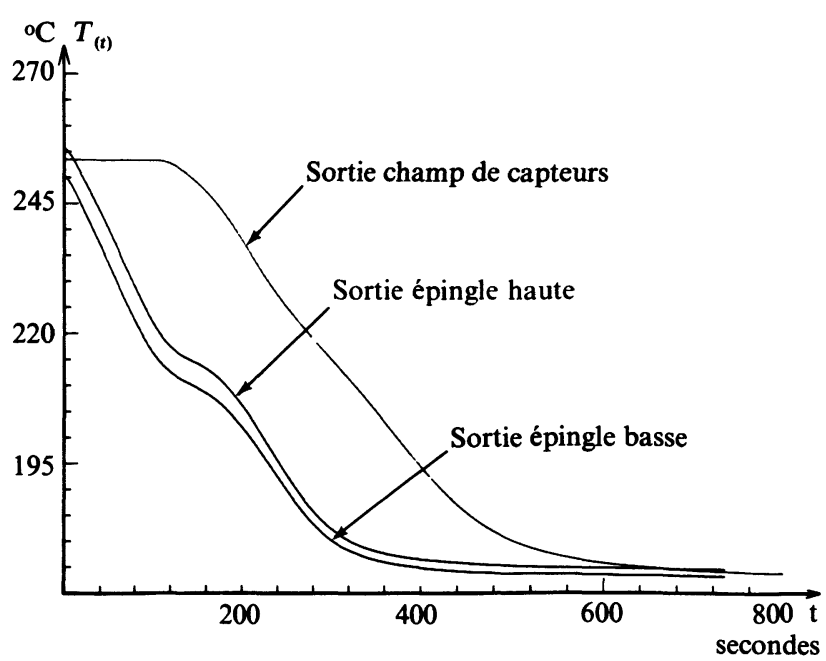

Fig. 6. - Réponse expérimentale du système et des soussystèmes.

[Experimental response of system and sub-systems.]

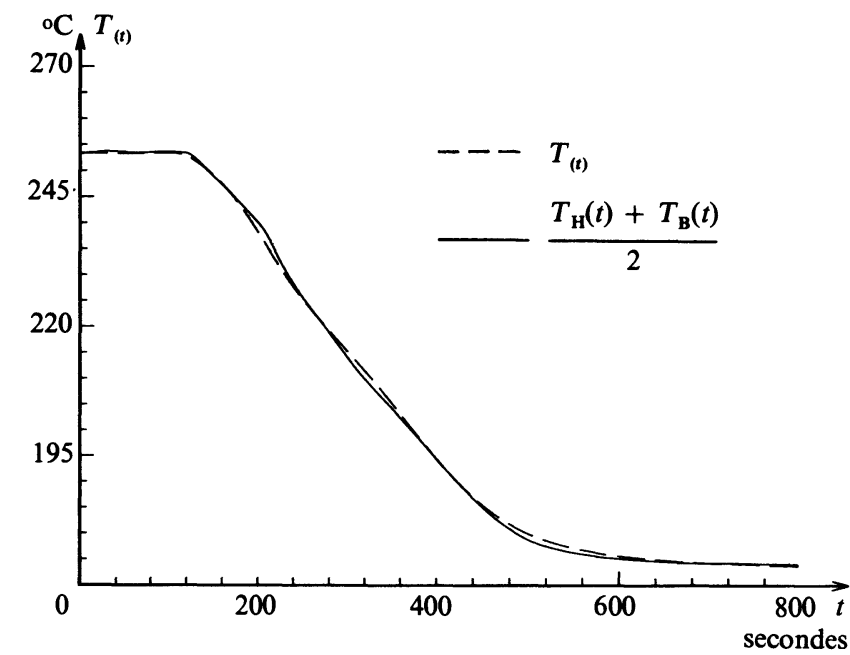

Fig. 6bis. - Réponse globale approchée par la demi-somme des réponses des sous-systèmes.

[Global response approximated by the half sum of subsystems responses.]

systèmes (épingles hautes et basses) car elle traduit bien la structure du système.

A ce stade on peut se proposer de modéliser correctement chacune des deux épingles. Nous ne le ferons pas pour trois raisons :

i) $\mathrm{Vu}$ l'allure des réponses d'épingles, il faudrait les décomposer elles-mêmes en sous-systèmes (chaudières) et on aboutirait à un modèle plus complexe.

ii) Alors que les températures de sortie d'épingle seraient correctement modélisées il ne serait pas du tout tenu compte des canalisations (en dehors du retard).

iii) La relation (1) n'est certainement pas tout à fait exacte; par exemple les débits dépendant de la tempé- 
rature ne sont pas généralement égaux et donc la moyenne arithmétique ne convient pas.

Nous préférons modéliser des épingles virtuelles (sous-systèmes haut et bas) dont la dynamique est notablement différente de celle des épingles réelles, mais qui prennent en compte les épingles et les canalisations. Il est clair que ces canalisations, non seulement introduisent des temps de retard, mais également modifient les temps de réponse. Quoiqu'il en soit ces épingles virtuelles donnent grâce à la relation (1), une bonne représentation de la température de sortie du champ.

\section{Premier modèle.}

4.1 Hypothìses. - Le système est décomposable en deux épingles virtuelles qui ont :

i) les mêmes états d'équilibre (par exemple $T_{\mathrm{f}}, T_{\mathrm{d}}$ ),

ii) des dynamiques différentes, représentables chacune par une seule constante de temps.

Remarque : Les longueurs différentes des canalisations justifient d'attribuer une dynamique différente aux épingles, celles du bas ayant une constante de temps plus grande (signal plus amorti car temps de parcours plus long).

4.2 INTERPRÉTATION PHYSIQUE DU MODĖLE. - Chaque épingle est représenté par une E.L.O.D.I.E. du premier ordre, ainsi par exemple :

$$
\dot{T}_{\mathrm{B}}=-\alpha_{\mathrm{B}}\left(T_{\mathrm{B}}-T_{\mathrm{d}}\right)+a_{\mathrm{B}} \Phi\left(t-\tau_{\mathrm{B}}\right)
$$

où $\Phi$ est le flux solaire mesuré.

Les coefficients $\alpha_{\mathrm{B}}, T_{\mathrm{d}}, \tau_{\mathrm{B}}$ seront à identifier; pour avoir le coefficient du flux il suffit de se placer à l'état d'équilibre $T_{\mathrm{B}}=T_{\mathrm{f}}, \Phi=\Phi_{0}$

$$
a_{\mathrm{B}}=\alpha_{\mathrm{B}} \frac{\left(T_{\mathrm{f}}-T_{\mathrm{d}}\right)}{\Phi_{0}} .
$$

Remarquons que le temps de retard est inclus dans le flux : on a donc une formulation compacte et complète.

Du point de vue phénoménologique, cela revient à dire que l'épingle est représentée comme un "compartiment " (réservoir) homogène en température, traversé par un fluide de débit volumique $q$, de température d'entrée $T_{e}$, de température de sortie $T_{\mathrm{B}}$.

Ce réservoir reçoit de l'extérieur un flux énergétique proportionnel à $\Phi: \eta \Phi$ et réémet un flux proportionnel à $\left(T_{\mathrm{B}}-T_{\mathrm{a}}\right): k\left(T_{\mathrm{B}}-T_{\mathrm{a}}\right)$ où $T_{\mathrm{a}}$ est la température du milieu ambiant.

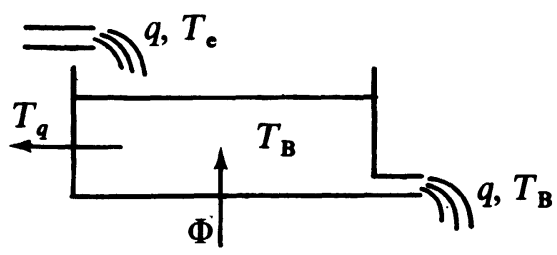

Le bilan énergétique conduit à :

$$
\begin{aligned}
m c q T_{\mathrm{B}}=\left[-q c\left(T_{\mathrm{B}}-T_{\mathrm{e}}\right)-k\left(T_{\mathrm{B}}-T_{\mathrm{a}}\right)\right. & + \\
& \left.+\eta \Phi\left(t-\tau_{\mathrm{B}}\right)\right] \mathrm{d} t .
\end{aligned}
$$

Où $c$ est la chaleur massique de fluide caloporteur et $m$ la masse de fluide dans le réservoir, ce qui justifie l'équation (2) avec par identification :

$$
\begin{aligned}
& \alpha_{\mathrm{B}}=\frac{q}{m}+\frac{k}{m c} \\
& T_{\mathrm{d}}=\frac{1}{\alpha_{\mathrm{B}}}\left[\frac{q}{m} T_{\mathrm{e}}+\frac{k}{m c} T_{\mathrm{a}}\right] \\
& a_{\mathrm{B}}=\frac{\eta}{m c} .
\end{aligned}
$$

Cette approche physique permet d'accéder aux lois reliant les coefficients du modèle $\left(\alpha_{\mathrm{B}}, T_{\mathrm{d}}, a_{\mathrm{B}}\right)$ aux paramètres de la structure $\left(q, T_{\mathrm{e}}, T_{\mathrm{a}}\right)$. On remarque par exemple que la valeur propre $\alpha_{B}$ dépend linéairement du débit. Pour avoir précisément ces lois, il suffit de réaliser deux expériences à débit différent.

Le champ est donc représenté par :

$$
T=\frac{1}{2}\left(T_{\mathrm{H}}+T_{\mathrm{B}}\right) .
$$

Modèle 1.

$$
\begin{aligned}
& \dot{T}_{\mathrm{H}}=-\alpha_{\mathrm{H}}\left(T_{\mathrm{H}}-T_{\mathrm{d}}\right)+a_{\mathrm{H}} \Phi\left(t-\tau_{\mathrm{H}}\right) \\
& \dot{T}_{\mathrm{B}}=-\alpha_{\mathrm{B}}\left(T_{\mathrm{B}}-T_{\mathrm{d}}\right)+a_{\mathrm{B}} \Phi\left(t-\tau_{\mathrm{B}}\right) .
\end{aligned}
$$

4.3 IDENTIFICATION Des Paramètres. - On identifie la réponse expérimentale (Fig. 1) à la réponse théorique du modèle, ce qui nous permet d'obtenir les paramètres.

On cherche donc les réponses sous la forme :

$T_{\mathrm{B}}(t)=T_{\mathrm{f}}+\Gamma\left(t-\tau_{\mathrm{B}}\right)\left[A \exp \left(-\alpha_{\mathrm{B}}\left(t-\tau_{\mathrm{B}}\right)\right)+B\right]$

$T_{\mathrm{H}}(t)=T_{\mathrm{f}}+\Gamma\left(t-\tau_{\mathrm{H}}\right)\left[C \exp \left(-\alpha_{\mathrm{H}}\left(t-\tau_{\mathrm{H}}\right)\right)+D\right]$

avec les conditions aux limites

$$
\begin{aligned}
T_{\mathrm{B}}\left(\tau_{\mathrm{B}}\right) & =\tau_{\mathrm{H}}\left(\tau_{\mathrm{H}}\right)=T_{\mathrm{f}} \\
T_{\mathrm{B}}(+\infty) & =T_{\mathrm{H}}(+\infty)=T_{\mathrm{d}}
\end{aligned}
$$

qui donnent :

$$
A=C=-B=-D=\left(T_{\mathrm{f}}-T_{\mathrm{d}}\right)
$$

Ainsi :

$$
\begin{aligned}
T(t)=T_{\mathrm{f}}+ & \frac{\left(T_{\mathrm{f}}-T_{\mathrm{d}}\right)}{2}\left[\Gamma\left(t-\tau_{\mathrm{H}}\right) \times\right. \\
& \times\left(\exp \left(-\alpha_{\mathrm{H}}\left(t-\tau_{\mathrm{H}}\right)\right)-1\right) \\
& \left.+\Gamma\left(t-\tau_{\mathrm{B}}\right)\left(\exp \left(-\alpha_{\mathrm{B}}\left(t-\tau_{\mathrm{B}}\right)\right)-1\right)\right] .
\end{aligned}
$$

Considérons la partie extrême droite du graphe $T(t)$; puisque l'on a vu qu'elle était composée d'une 
seule exponentielle, il s'agit alors, d'après nos hypothèses, de la réponse des épingles basses (la réponse des épingles hautes étant déjà amortie).

On a :

$$
\begin{aligned}
T(t)=T_{\mathrm{f}}-\frac{\left(T_{\mathrm{f}}-T_{\mathrm{d}}\right)}{2}+\frac{\left(T_{\mathrm{f}}-T_{\mathrm{d}}\right)}{2} \times \\
\times\left[\exp \left(\alpha_{\mathrm{B}} \tau_{\mathrm{B}}\right) \exp \left(-\alpha_{\mathrm{B}} t\right)-1\right] \\
T(t)=T_{\mathrm{d}}+\left[\frac{\left(T_{\mathrm{f}}-T_{\mathrm{d}}\right)}{2} \exp \left(\alpha_{\mathrm{B}} \tau_{\mathrm{B}}\right)\right]\left(\exp \left(-\alpha_{\mathrm{B}} t\right)\right) .
\end{aligned}
$$

On obtient donc, grâce à la figure 2 , en prenant la pente de la droite :

$\alpha_{\mathrm{B}}=\alpha$

$\tau_{\mathbf{B}}$ est déterminé directement sur la courbe.

Restent à déterminer $\tau_{\mathrm{H}}$ et $\alpha_{\mathrm{H}}$. Le temps de retard des épingles hautes est forcément le même que celui du champ total; on a donc $\tau_{\mathbf{H}}$ par mesure directe du décalage entre le flux solaire et la réponse $T(t)$. Pour obtenir $\alpha_{H}$ on va se placer dans l'intervalle de temps compris entre $\tau_{\mathbf{H}}$ et $\tau_{\mathbf{B}}$. Alors :

$T(t)=T_{\mathrm{f}}+\frac{\left(T_{\mathrm{f}}-T_{\mathrm{d}}\right)}{2}\left[\exp \left(-\alpha_{\mathrm{H}}\left(t-\tau_{\mathrm{H}}\right)\right)-1\right]$

$T(t)=\frac{\left(T_{\mathrm{f}}+T_{\mathrm{d}}\right)}{2}+\frac{\left(T_{\mathrm{f}}-T_{\mathrm{d}}\right)}{2}\left[\exp \left(-\alpha_{\mathrm{H}}\left(t-\tau_{\mathrm{H}}\right)\right)\right]$.

Par conséquent $\log \left(T-\frac{\left(T_{\mathrm{f}}+T_{\mathrm{d}}\right)}{2}\right)$ devrait être linéaire dans la zone $\left(\tau_{\mathrm{H}}, \tau_{\mathrm{B}}\right)$. Or la figure 7 montre clairement qu'il n'en est rien, ce qui veut dire que nos hypothèses ne sont pas correctes. Néanmoins on peut prendre une approximation linéaire moyenne, qui fournit $\alpha_{H}$. Les réponses individuelles des épingles virtuelles sont représentées figure 7 bis et l'on voit figure 8

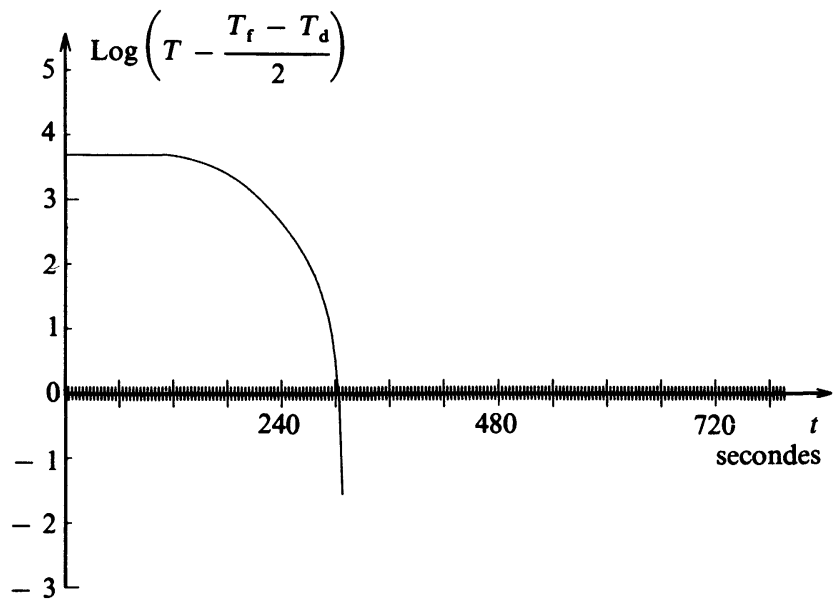

Fig. 7. - Recherche de $\alpha_{H}$ par tracé logarithmique.

[Determination of $\alpha_{\mathbf{H}}$ using a logarithmic plot.]

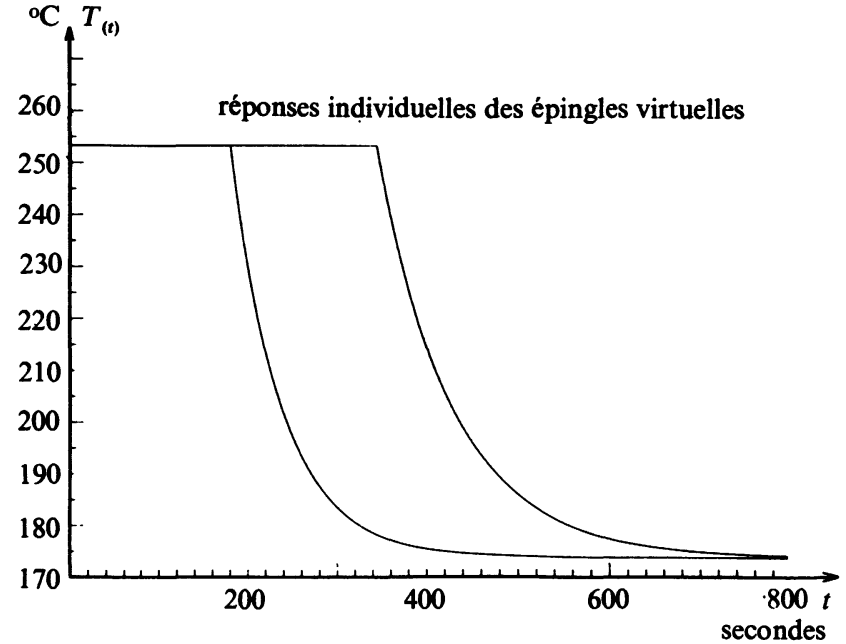

Fig. 7bis. - Réponse des sous-systèmes virtuels du premier modèle.

[Response of virtual sub-sytems (first model).]

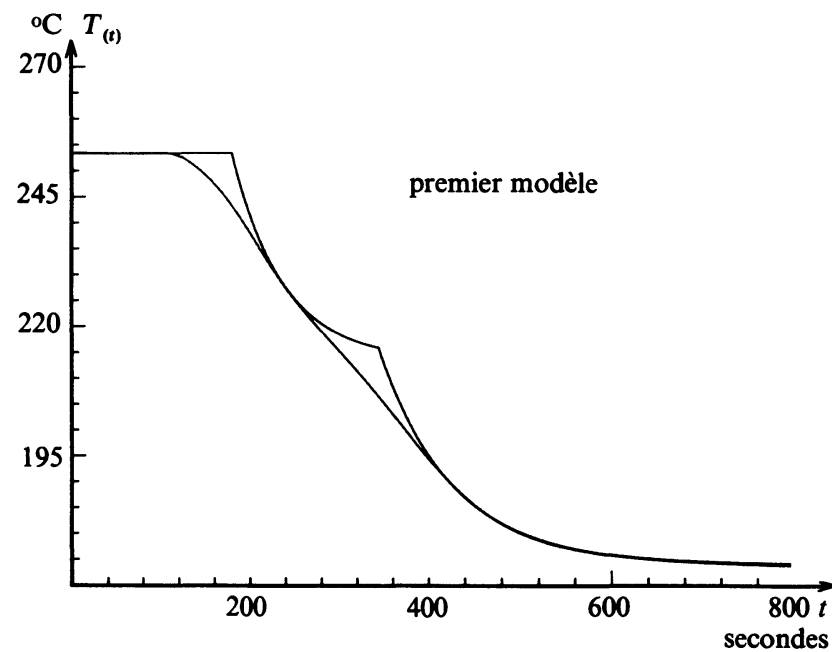

Fig. 8. - Comparaison entre la réponse réelle du champ et celle du premier modèle.

[Comparaison between actual and computed response (first model).]

que l'approche de $T(t)$ par cette voie est relativement satisfaisante.

Remarques :

1. Quelles que soient les variations du flux solaire, et pour un débit et une température d'entrée constants, on a la loi de comportement de la température de sortie du champ.

2. Les épingles virtuelles ne correspondent pas aux épingles réelles sauf pour les positions d'équilibre. Comme l'on a besoin de valeurs initiales $T_{\mathrm{H}}(0), T_{\mathrm{B}}(0)$ et que l'on ne peut les obtenir qu'à partir des températures mesurées $T_{1}, T_{3}$, il faut choisir comme instant initial un moment où le champ est en état d'équilibre. 
4.4 PREMIER TEST : REFOCAlisation DU CHAMP. -

Le champ étant totalement défocalisé et en équilibre : $T_{\mathrm{d}}$, on la refocalise avec un flux solaire approximativement constant : on arrive à une valeur d'équilibre $T_{\mathrm{f}}$. Il faut noter que $T_{\mathrm{d}}$ dépend des paramètres $q, T_{\mathrm{e}}, T_{\mathrm{a}}$. Pour ce modèle, nous n'avons pas déterminé les coefficients des lois (3); aussi nous nous contentons de réajuster $T_{\mathrm{d}}$ quand on passe d'une expérience à l'autre. De plus, la situation étant simple $\left(T_{\mathrm{d}}\right.$ et $\Phi$ constants) il est inutile de se servir des formules générales d'intégration. Il suffit d'écrire la forme des solutions :

$T_{\mathrm{H}}(t)=T_{\mathrm{d}}+\Gamma\left(t-\tau_{\mathrm{H}}\right)\left[A \exp \left(-\alpha_{\mathrm{H}}\left(t-\tau_{\mathrm{H}}\right)\right)+B\right]$

avec les conditions $\quad T_{\mathrm{H}}\left(\tau_{\mathrm{H}}\right)=T_{\mathrm{d}} \rightarrow A+B=0$

$$
T_{\mathrm{H}}(\infty)=T_{\mathrm{f}} \rightarrow T_{\mathrm{d}}+B=T_{\mathrm{f}}
$$

avec

$$
\begin{gathered}
\alpha_{\mathrm{H}}\left(T_{\mathrm{f}}-T_{\mathrm{d}}\right)=a_{\mathrm{H}} \phi \\
T_{\mathrm{f}}=T_{\mathrm{d}}+\frac{a_{\mathrm{H}}}{\alpha_{\mathrm{H}}} \Phi .
\end{gathered}
$$

Ainsi :

$$
\begin{aligned}
T_{\mathrm{H}}(t)=T+\Gamma\left(t-\tau_{\mathrm{H}}\right) & \left(T_{\mathrm{f}}-T\right) \times \\
\times & \times\left[1-\exp \left(-\alpha_{\mathrm{H}}\left(t-\tau_{\mathrm{H}}\right)\right)\right]
\end{aligned}
$$

et une formule analogue pour $T_{\mathrm{B}}$. On voit sur la figure 9 que le résultat est convenable.

Premier test : focalisation du champ de capteurs

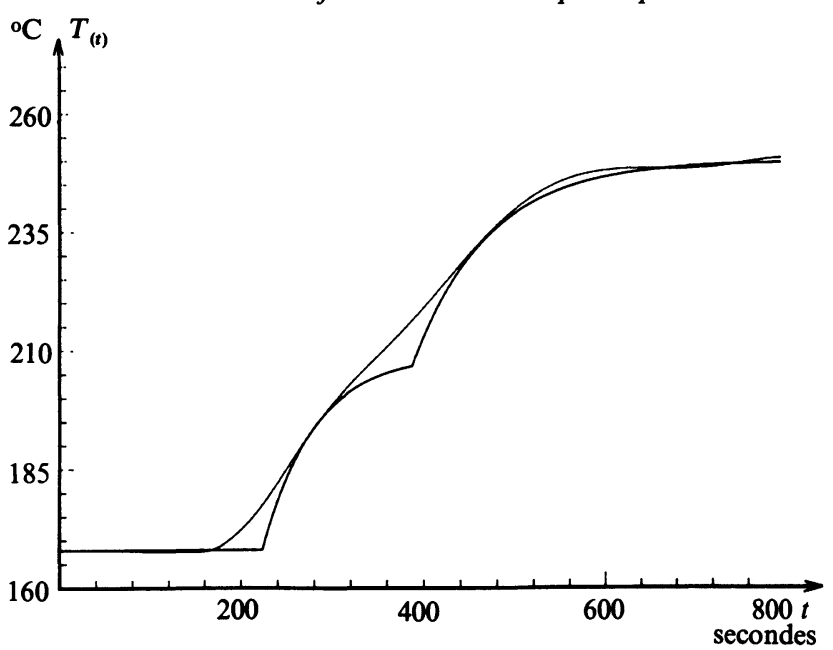

Fig. 9. - Premier test d'efficacité du premier modèle : réponse à une refocalisation totale du champ.

[First validation test of the first model : response to the focalization of the whole field.]

4.5 DEUXIÈME TEST : REFOCALISATION DE LA MOITIÉ DU CHAMP. - On réalise l'expérience suivante : on défocalise une épingle du haut et une épingle du bas (les autres restant focalisées); une fois l'équilibre atteint on refocalise ces deux épingles. Le flux solaire est resté sensiblement constant durant la durée de l'expérience.

Les deux épingles du haut sont régies par la même équation (4) où l'on remplace successivement $T_{\mathrm{H}}$ par $T_{1}$ et $T_{2}$. La seule différence pour les deux épingles c'est que l'une va être toujours soumise au flux $\phi_{0}$ alors que l'autre va subir un échelon de flux de zéro à $\Phi_{0}$. Etant donné l'identité et la linéarité des équations, cela revient à dire que $T_{\mathrm{H}}$, qui est égale à la demisomme de $T_{1}$ et $T_{2}$, obéit à l'équation (4) dans laquelle le flux subit un échelon de $\phi_{0} / 2$ à $\phi_{0}$ avec une température initiale d'équilibre :

$$
T_{\mathrm{H}}(0)=\frac{1}{2}\left[T_{1}(0)+T_{2}(0)\right]=\frac{1}{2}\left(T_{\mathrm{d}}+T_{\mathrm{f}}\right) .
$$

On a ainsi :

$$
\begin{array}{cc}
T_{\mathrm{H}}=\frac{\left(T_{\mathrm{d}}+T_{\mathrm{f}}\right)}{2}+ & \Gamma\left(t-\tau_{\mathrm{H}}\right) \times \\
& \times\left[A \exp \left(-\alpha_{\mathrm{H}}\left(t-\tau_{\mathrm{H}}\right)\right)+B\right] \\
\text { avec } & A+B=0 ;
\end{array}
$$

$$
T_{\mathrm{f}}=B+\frac{\left(T_{\mathrm{d}}+T_{\mathrm{f}}\right)}{2}
$$

d'où :

$$
\begin{aligned}
T=\frac{\left(T_{\mathrm{d}}+T_{\mathrm{f}}\right)}{2}+\frac{\left(T_{\mathrm{f}}-T_{\mathrm{d}}\right)}{2} \times \\
\quad \times\left[\Gamma\left(t-\tau_{\mathrm{H}}\right)\left(1-\exp \left(-\alpha_{\mathrm{H}}\left(t-\tau_{\mathrm{H}}\right)\right)\right)\right. \\
\left.+\Gamma\left(t-\tau_{\mathrm{H}}\right)\left(1-\exp \left(-\alpha\left(t-\tau_{\mathrm{B}}\right)\right)\right)\right] .
\end{aligned}
$$

Là encore on constate, figure 10 , un accord satisfaisant entre la courbe théorique et la courbe expérimentale.

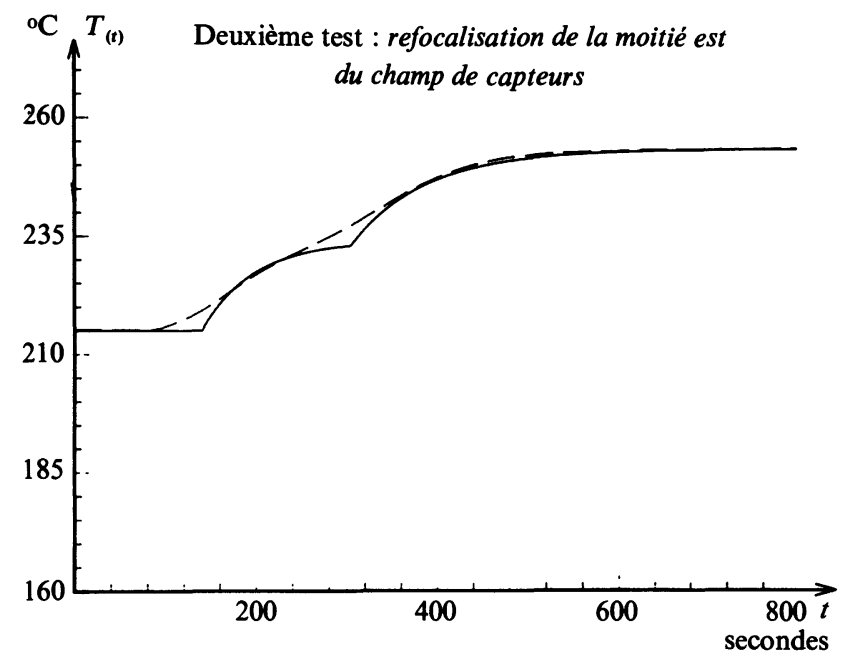

Fig. 10. - Deuxième test d'efficacité du premier modèle : réponse à la refocalisation d'une moitié du champ.

[Second validation test of the first model : response to the focalization of the half-field.] 


\section{Deuxième modèle.}

5.1 Hypothèses. - Le modèle précédent, qui ne traduit déjà pas fidèlement le comportement du champ, présente deux inconvénients importants : d'une part il fait jouer aux épingles haut et bas des rôles très différents, ce qui n'est physiquement pas justifié; d'autre part il ne conduit pas à une détermination systématique des coefficients (en particulier $\alpha_{\mathrm{H}}$ ), ce qui ne fait que traduire une inadéquation à la réalité.

Nous partons maintenant avec les hypothèses suivantes :

* les épingles ont des réponses identiques;

* leur dynamique se traduit par deux constantes de temps.

5.2 INTERPRÉTATION PHYSIQUE DU MODÈLE. - Les sous-systèmes haut et bas, sont caractérisés par deux valeurs propres : $\alpha$ et $\beta$; ils sont donc représentables par une E.L.O.D.I.E. du second ordre :

$$
\begin{aligned}
& \dddot{T}_{\mathrm{H}}+(\alpha+\beta) \dot{T}_{\mathrm{H}}+\alpha \beta\left(T_{\mathrm{H}}-T_{\mathrm{d}}\right)=a \Phi\left(t-\tau_{\mathrm{H}}\right) \\
& \ddot{T}_{\mathrm{B}}+(\alpha+\beta) \dot{T}_{\mathrm{B}}+\alpha \beta\left(T_{\mathrm{B}}-T_{\mathrm{d}}\right)=a \Phi\left(t-\tau_{\mathrm{B}}\right)
\end{aligned}
$$

et la relation fonctionnelle reliant les deux sous-systèmes étant toujours :

$$
T=\frac{1}{2}\left(T_{\mathrm{H}}+T_{\mathrm{B}}\right) .
$$

Ces équations admettent bien (en régime libre : $\Phi=0$ ) $\alpha$ et $\beta$ comme valeurs propres et $T_{\mathrm{d}}$ comme valeur limite; le coefficient a est donné par :

$$
a=\alpha \beta \frac{\left(T_{\mathrm{f}}-T_{\mathrm{d}}\right)}{\Phi} .
$$

On voit qu'en fait, le système entier est représentable par une E.L.O.D.I.E. du second ordre :

$$
\begin{aligned}
\dddot{T}+(\alpha+\beta) \dot{T}+ & \alpha \beta\left(T-T_{\mathrm{d}}\right)= \\
& =\frac{a}{2}\left[\Phi\left(t-\tau_{\mathrm{H}}\right)+\Phi\left(t-\tau_{\mathrm{B}}\right)\right] .
\end{aligned}
$$

On peut donner une interprétation phénoménologique de cette équation par une représentation à deux réservoirs (puisqu'il y a deux valeurs propres) pour lesquelles on fait le bilan thermique :

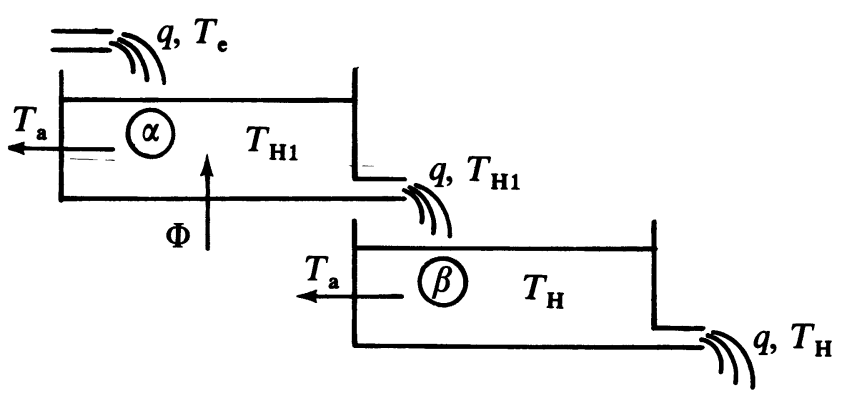

$$
\begin{aligned}
& m_{1} c q d T_{\mathrm{H} 1}= \\
& \quad=\left[-q c\left(T_{\mathrm{H} 1}-T_{\mathrm{e}}\right)-K_{1}\left(T_{\mathrm{H} 1}-T_{\mathrm{a}}\right)+\eta \Phi\right] \mathrm{d} t \\
& m_{2} c q d T_{\mathrm{H}}=\left[-q c\left(T_{\mathrm{H}}-T_{\mathrm{H} 1}\right)-K_{2}\left(T_{\mathrm{H}}-T_{\mathrm{a}}\right)\right] \mathrm{d} t .
\end{aligned}
$$

Les notations étant analogues à celles du premier modèle.

Soit sous forme matricielle :

$$
\left[\begin{array}{l}
\dot{T}_{\mathrm{H} 1} \\
\dot{T}_{\mathrm{H}}
\end{array}\right]=\left[\begin{array}{cc}
-\alpha & 0 \\
C_{1} & -\beta
\end{array}\right]\left[\begin{array}{l}
T_{\mathrm{H} 1} \\
T_{\mathrm{H}}
\end{array}\right]+\left[\begin{array}{l}
C_{2}+a \Phi \\
C_{3}
\end{array}\right]
$$

qui par diagonalisation conduit à (5); (où les $C_{i}$ sont des constantes intermédiaires).

Cette interprétation laisse à penser que le premier réservoir pourrait représenter l'épingle proprement dite et le deuxième la canalisation d'amenée.

5.3 IDENTIFICATION DES PARAMÈTRes. - Pour représenter l'essai fondamental (défocalisation totale du champ) on écrit :

$$
\begin{aligned}
T_{\mathrm{H}}=T_{\mathrm{f}}+\Gamma\left(t-\tau_{\mathrm{H}}\right) & {\left[A \exp \left(-\alpha\left(t-\tau_{\mathrm{H}}\right)\right)+\right.} \\
+ & \left.B \exp \left(-\beta\left(t-\tau_{\mathrm{H}}\right)\right)+C\right]
\end{aligned}
$$

idem pour $T_{\mathrm{B}}$.

Nous avons donc 7 coefficients à déterminer : $A, B, C, \alpha, \beta, \tau_{\mathrm{H}}, \tau_{\mathrm{B}}$. En fait, comme nous l'avons déjà dit, $\tau_{\mathbf{H}}$ est immédiatement déterminé. Nous avons introduit un deuxième temps de réponse essentiellement pour réduire les angles saillants qui apparaissaient dans le premier modèle et donc pour assurer un début de courbe à pente nulle. Aussi nous pouvons écrire :

$$
\left.\begin{array}{l}
\dot{T}_{\mathrm{H}}\left(\tau_{\mathrm{H}}\right)=0 \\
T_{\mathrm{H}}\left(\tau_{\mathrm{H}}\right)=T_{\mathrm{f}} \\
T_{\mathrm{H}}(\infty)=T_{\mathrm{d}}
\end{array}\right] \rightarrow\left[\begin{array}{l}
\alpha A+\beta B=0 \\
A+B+C=0 \\
C=T_{\mathrm{d}}-T_{\mathrm{f}} .
\end{array}\right.
$$

Il reste donc 5 inconnues $A, B, \alpha, \beta, \tau_{\mathrm{B}}$ et nous disposons de deux équations :

$$
\begin{aligned}
\alpha A+\beta B & =0, \\
A+B & =T_{\mathrm{f}}-T_{\mathrm{d}} .
\end{aligned}
$$

Restent à trouver 3 équations. Pour cela considérons d'abord la partie extrême droite de la figure 1 ; les exponentielles en $\beta$ y sont déjà amorties (puisqu'on ne trouve plus qu'une seule exponentielle) et l'on a :

$$
\begin{aligned}
T(t) & =T_{\mathrm{d}}+\frac{A}{2}\left[\exp \left(-\alpha\left(t-\tau_{\mathrm{H}}\right)\right)+\exp \left(-\alpha\left(t-\tau_{\mathrm{B}}\right)\right)\right] \\
& =T_{\mathrm{d}}+\frac{A}{2}\left[1+\exp \left(\alpha\left(\tau_{\mathrm{B}}-\tau_{\mathrm{H}}\right)\right)\right]\left(\exp \left(-\alpha\left(t-\tau_{\mathrm{H}}\right)\right)\right) .
\end{aligned}
$$

D'où grâce à la figure 2 on obtient $\alpha$ et le coefficient multiplicatif qui fournit $\tau_{\mathbf{B}}$ en fonction de $A$. La dernière équation manquante est obtenue en considérant le développement limité de $T(t)$ au voisinage de $t=\tau_{\mathrm{H}}$. 


$$
\begin{aligned}
T(t)=\frac{1}{2}\left(T_{\mathrm{d}}+T_{\mathrm{f}}+A+B\right) & -\frac{1}{2}(\alpha A+\beta B)\left(t-\tau_{\mathrm{H}}\right)+ \\
& +\frac{1}{4}\left(A \alpha^{2}+B \beta^{2}\right)\left(t-\tau_{\mathrm{H}}\right)^{2}+\cdots \\
= & T_{\mathrm{f}}+\gamma\left(t-\tau_{\mathrm{H}}\right)^{2}+\cdots .
\end{aligned}
$$

Ainsi

$$
A \alpha^{2}+B \beta^{2}=4 \gamma
$$

$\gamma$ étant la pente de la courbe $T$ en fonction de $\left(t-\tau_{H}\right)^{2}$ pour $t$ légèrement supérieur à $\tau_{\mathrm{H}}$ : figure 11 .

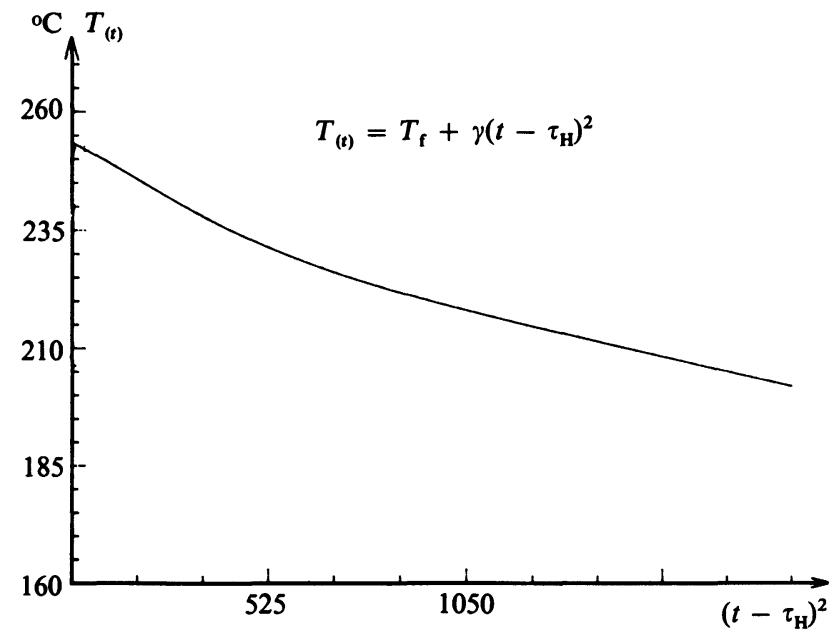

Fig. 11. - Obtention de la dérivée seconde à l'origine.

[Obtention of the second derivative at the origin.]

Il suffit de résoudre le système algébrique :

$$
\begin{aligned}
& A+B=T_{\mathrm{f}}-T_{\mathrm{d}} \\
& A+B \frac{\beta}{\alpha}=0 \\
& A+B\left(\frac{\beta}{\alpha}\right)^{2}=\frac{4 \gamma}{\alpha^{2}} .
\end{aligned}
$$

Ce qui donne $: \beta=\frac{4 \gamma}{\alpha\left(T_{\mathrm{f}}-T_{\mathrm{d}}\right)}$

d'où ensuite $B$ puis $A$.

On a ainsi une méthode systématique d'obtention des coefficients. Avec les valeurs ainsi obtenues, la figure 12 montre que le comportement des épingles virtuelles est très différent de celui des épingles réelles; mais l'on voit, figure 13 que l'on arrive à une approche très correcte $\mathrm{du}$ comportement global du champ. Seule la zone centrale est mal approchée, ce qui n'est pas étonnant puisque nous n'avons écrit de conditions que pour le début ou la fin de la courbe.

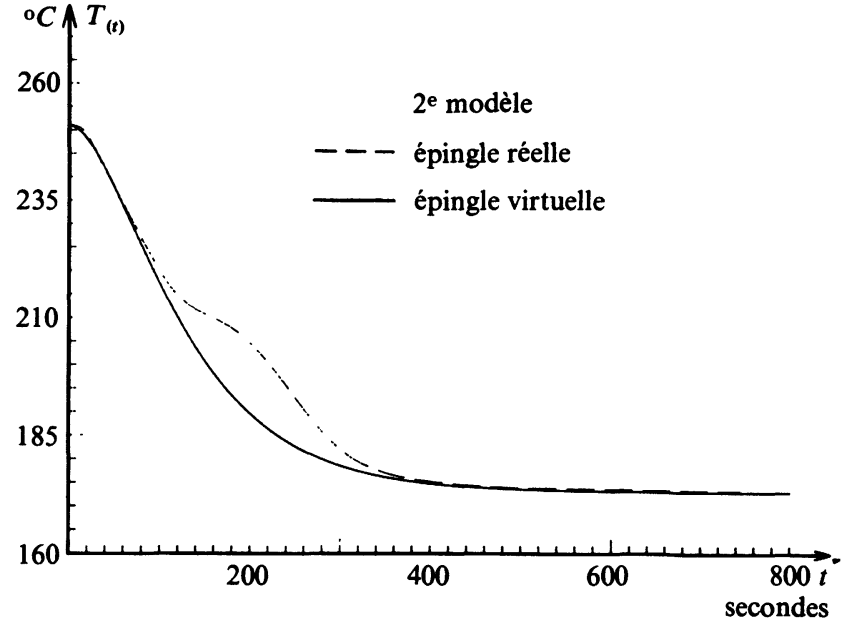

Fig. 12. - Comportement des sous-systèmes réels et des sous-systèmes virtuels ( $2^{\mathrm{e}}$ modèle).

[Comparison between actual and virtual sub-systems (second model).]

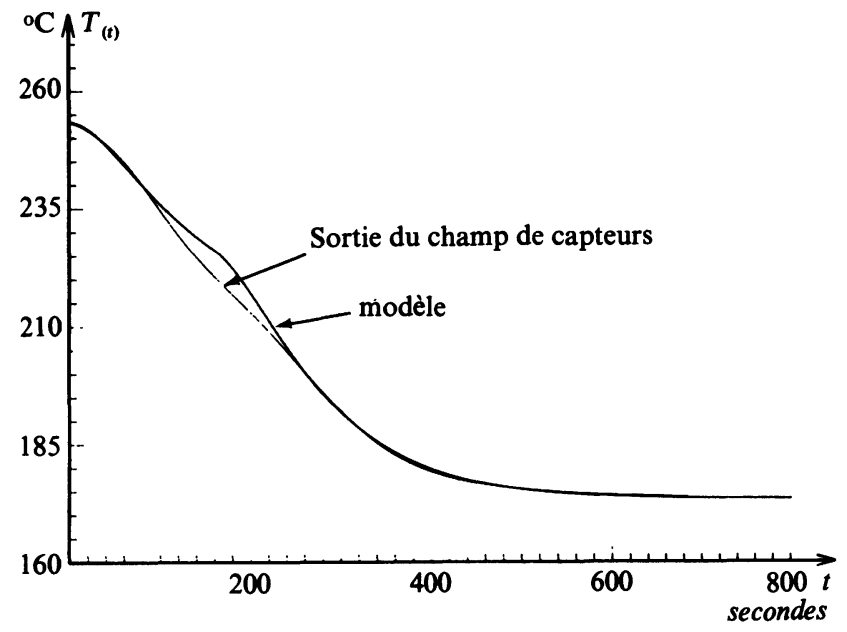

Fig. 13. - Comparaison entre la réponse réelle du champ et celle du deuxième modèle.

[Comparison between actual and computed response (second model).]

\section{Valeurs numériques}

$$
\begin{gathered}
A=227 ; \quad B=-147 ; \quad \alpha=0,192 \mathrm{~s}^{-1} \\
\beta=0,296 \mathrm{~s}^{-1} ; \quad \tau_{\mathrm{B}}-\tau_{\mathrm{H}}=160 \mathrm{~s} \\
T_{\mathrm{d}}=173,3{ }^{\circ} \mathrm{C} ; \quad T_{\mathrm{f}}=253,3{ }^{\circ} \mathrm{C} .
\end{gathered}
$$

5.4 TestS DE validité. - Nous avons testé ce second modèle sur trois expériences simples : les deux expériences déjà utilisées en 4.4 et 4.5 plus celle consistant à défocaliser une moitié du champ (une épingle haute et une basse). 
Dans chaque cas les températures ont la forme :

$$
\begin{aligned}
T_{\mathrm{H}}(t)=D+\Gamma\left(t-\tau_{\mathrm{H}}\right)[ & A \exp \left(-\alpha\left(t-\tau_{\mathrm{H}}\right)\right)+ \\
& \left.+B \exp \left(-\beta\left(t-\tau_{\mathrm{H}}\right)\right)+C\right]
\end{aligned}
$$

et les coefficients inconnus sont déterminés par :

$$
\begin{gathered}
D=T_{\mathbf{H}}\left(\tau_{\mathbf{H}}\right) \\
A+B+C=0 \\
C=T_{\mathbf{H}}(\infty)-D \\
\alpha A+\beta B=0 .
\end{gathered}
$$

Les figures $14,15,16$, qui comparent les courbes théoriques et expérimentales, montrent la validité

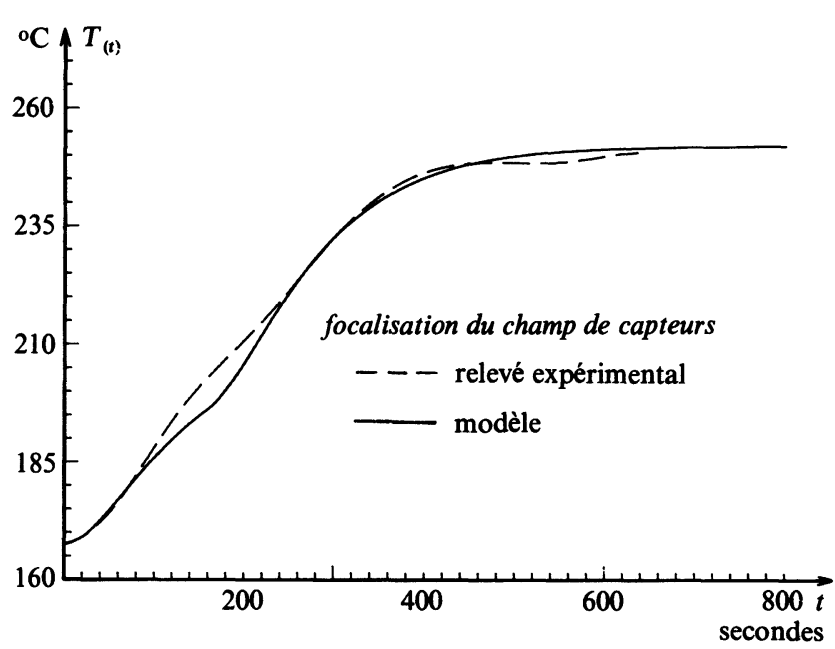

Fig. 14. - Premier test pour le deuxième modèle : refocalisation totale du champ.

[First validation test of the second model : focalisation of the whole field.]

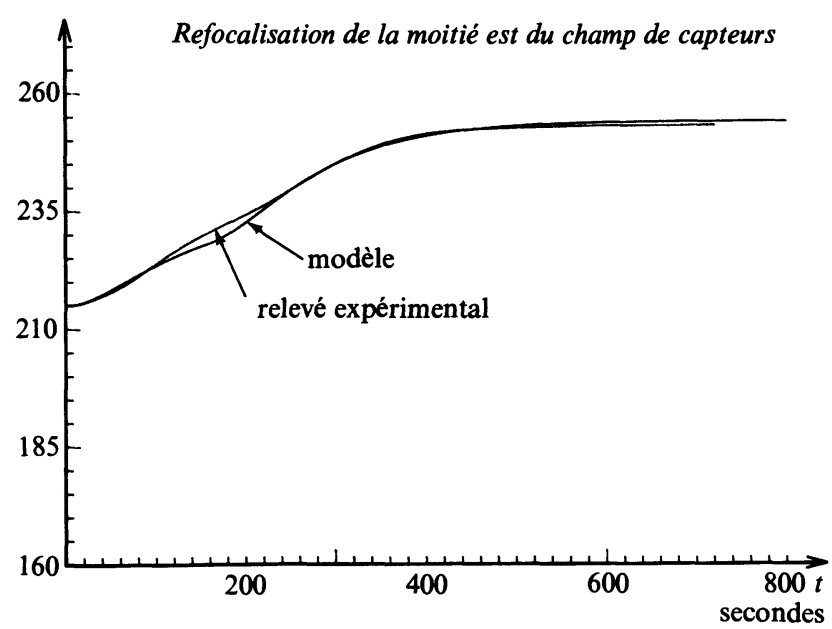

Fig. 15. - Deuxième test pour le deuxième modèle : demirefocalisation du champ.

[Second validation test of the second model : focalization of the half-field.]

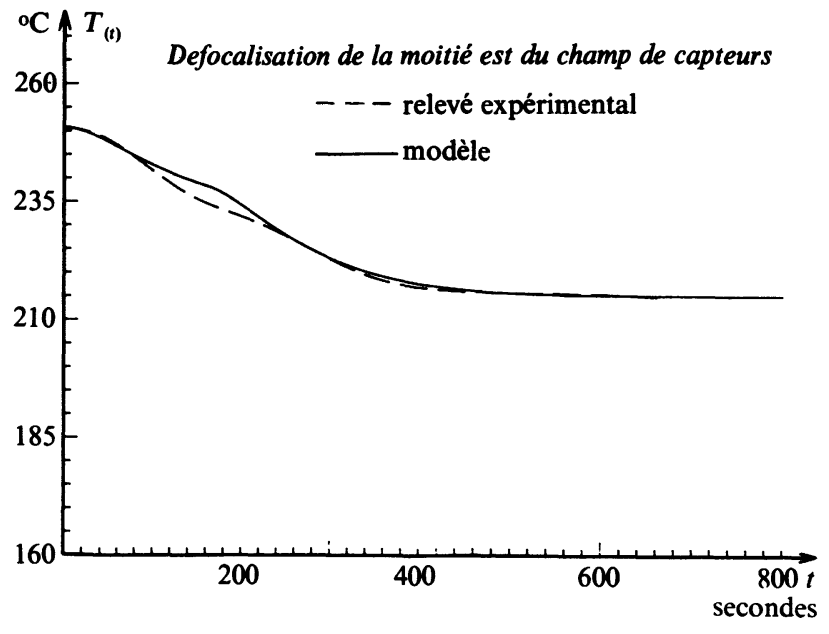

Fig. 16. - Troisième test pour le deuxième modèle : demidéfocalisation du champ.

[Third validation test of the second model : defocalisation of the half-field.]

de ce modèle, avec néanmoins toujours une erreur dans la zone centrale.

\section{Conclusion.}

Ce travail montre clairement l'efficacité de la démarche proposée, que nous rappelons.

0) On considère le champ de capteurs de la Centrale Solaire de Vignola, qui en régime de fonctionnement automatique régule la température de sortie par une commande rétroactive sur le débit de fluide.

1) On supprime cette commande : le débit devient constant et l'on travaille avec une température d'entrée également constante. On a un système passif qui va réagir en fonction des variations du seul flux solaire.

2) On réalise un échelon total de flux solaire (par défocalisation simultanée des huit chaudières) et l'on mesure la réponse du système, température de sortie/temps.

3) Le dépouillement de cet unique thermogramme par une méthode spectrale conduit très facilement à une équation différentielle linéaire :

$\ddot{T}+(\alpha+\beta) \dot{T}+\alpha \beta T=\frac{a}{2}\left[\Phi\left(t-\tau_{\mathbf{H}}\right)+\Phi\left(t-\tau_{\mathbf{B}}\right)\right]$.

Quelques expériences tests montrent que ce modèle très simple est en bon accord avec la réalité.

En somme une seule expérience, facile à réaliser, a permis de construire (avec des moyens numériques élémentaires) un modèle simple et précis d'un système aussi complexe que le Champ de Capteurs de Vignola. Bien sûr le modèle n'est valable que pour un débit et une température d'entrée nominaux. Néanmoins, en dehors de cette publication, nous avons construit le modèle pour différents débits $q$, et températures d'entrée $T_{\mathrm{e}}$ et obtenu les lois de dépendance des coeffi- 
cients de structure $\alpha, \beta, a, \tau_{\mathrm{H}}, \tau_{\mathrm{B}}$ par rapport aux paramètres $q, T_{\mathrm{e}}$. De là et connaissant les lois de variation, $q(t)$ et $T_{\mathrm{e}}(t)$, nous avons calculé (par intégration numérique) $T(t)$ et l'avons comparé, avec succès, à la réponse réelle du système sur des journées où le flux solaire, le débit et la température d'entrée variaient.

Pour ce système il n'est pas possible pour des raisons technico-économiques d'en modifier la structure. Enfin nous tentons de réaliser une commande prévisionnelle basée sur le raisonnement suivant :

l'entrée (le flux $\Phi$ ), la sortie (température $T$ ), les para- mètres de structure (débit $q$ température d'entrée $T_{\mathrm{e}}$ ) sont reliés par une relation fonctionnelle

$$
f\left(T, \Phi, T_{\mathrm{e}}, q\right)=0 .
$$

Le flux et la température d'entrée bien que non maîtrisables sont des fonctions connues du temps. La relation fonctionnelle $f$ fournit la loi $q(t)$ permettant de maîtriser $T(t)$.

La simplicité du modèle permet le calcul en temps réel de cette loi et par conséquent permet une commande prévisionnelle dynamique du champ.

\section{Bibliographie}

[1] Combes, J., Analyse comparative de centrales électrosolaires à collecteurs distribués. Thèse DI, Ecole Centrale des Arts et Manufactures, 1983.

[2] Riolacci, C., Contribution à l'étude des systèmes hélioénergétiques, Thèse DI, Institut National Polytechnique de Grenoble, 1985.

[3] BALbi, J. H., PerI, G., Modélisation dynamique des systèmes énergétiques à intrant solaire, Revue Gén. Ther. (1986).
[4] Balbi, J. H., Balbi, N., Modèle d'Etat d'un système énergétique solaire, Revue Gén. Ther. (1986).

[5] BOY-MARCOTTE, J. L., Les Centrales solaires de moyenne puissance à collecteurs distribués 100 à $1000 \mathrm{KWe}$, Société Bertin, Entropie 82 (1978).

[6] Bacconnet, Eynard-Machet, Prevost, Durniak, COSS, Capteur solaire à concentration. $\mathrm{XX}^{\mathrm{e}}$ Conférence Internationale COMPLES, Rabat, 1981. 